


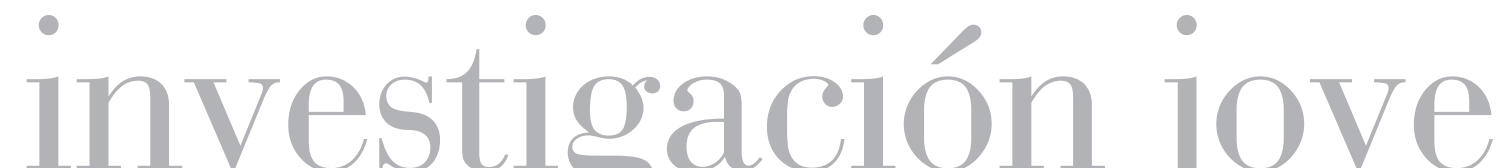

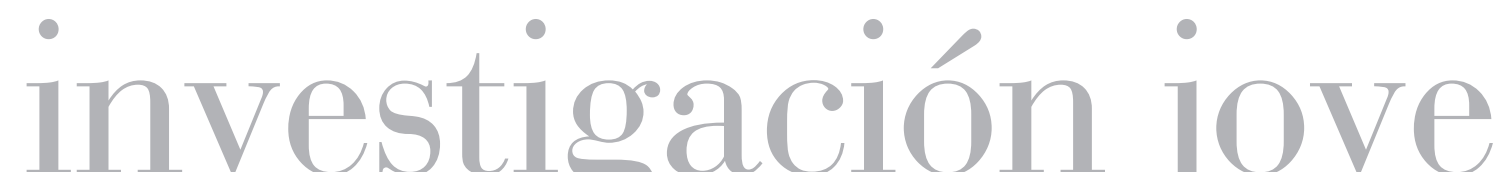





\title{
Problemas, actores y decisiones en las politicas públicas. Marco de análisis para el estudio de los programas de crédito educativo en Colombia ${ }^{1}$
}

\author{
Héctor Andrés Pérez Enciso ${ }^{2}$ \\ Universidad Nacional de Colombia, Bogotá, Colombia \\ hapereze@gmail.com \\ Recibido: 16 de febrero de 2016 \\ Aceptado: 25 de julio de 2016 \\ Disponible en línea: 20 de diciembre de 2016
}

1 El presente artículo constituye un trabajo de revisión, construido en el marco de la tesis de Maestría titulada: Evaluación del programa de Crédito ACCES y su transformación en la Linea de Crédito de Largo Plazo "Tú Elijes"; del ICETEX. Efectos de la transformación en las dinámicas de acceso a la educación superior por vía de los créditos educativos en Bogotá, 2013-2016 [en curso], para optar al título de Magíster en Evaluación y Aseguramiento de la Calidad de la Educación. Proyecto sin financiación.

2 Sociólogo, Universidad Nacional de Colombia. Estudios de Maestría en Evaluación y Aseguramiento de la Calidad de la Educación, Universidad Externado en Colombia. Profesional vinculado a la Dirección de Educación Media y Superior, Secretaría de Educación de Bogotá (SED) (Bogotá, Colombia). 


\title{
Problemas, actores y decisiones en las politicas públicas. Marco de análisis para el estudio de los programade crédito educativo en Colombia
}

\begin{abstract}
Resumen
Este artículo propone un marco de análisis para el estudio de los procesos decisionales asociados a la implementación de los créditos educativos en Colombia, como dispositivo de política pública destinado al fomento del acceso a la educación superior. El enfoque conceptual y la propuesta metodológica aqui presentados recogen elementos del paradigma de la complejidad y su pertinencia en el análisis de políticas públicas. Este marco de análisis se concentra en estudiar las relaciones existentes entre las corrientes de actores, problemas y decisiones de política, en cuya interrelación surgen elementos explicativos sobre la manera en que se dirige el proceso de making policy, desde la elaboración del problema hasta la toma de decisiones. El trabajo concluye con un esbozo acerca del modo en que la elaboración e implementación de los programas de crédito educativo en Colombia, impulsados por el Instituto Colombiano de Crédito Educativo y Estudios Técnicos en el Exterior (ICETEX), reúnen las condiciones para ser analizados desde la perspectiva conceptual y metodológica aquí propuesta.
\end{abstract}

Palabras clave: complejidad; políticas públicas; agenda de gobierno; problemas públicos; actores; proceso decisional

\section{Issues, Actors, and Decisions in Public Policy. Analytical Framework for the Study of Student Loan Programs in Colombia}

\begin{abstract}
This paper proposes an analytical framework for the study of decision-making processes associated with the implementation of student loans in Colombia, as a public policy device, aimed at promoting access to higher education. The conceptual approach and the methodology herein contain elements of the complexity paradigm and its relevance in public policy analysis. This framework of analysis focuses on studying the relationship between public policy actors, issues, and decisions, which reveals explanatory elements of the policy-making process, from the definition of the issue to the making of the decision. This paper concludes with an outline on how the development and implementation of student loan programs in Colombia, promoted by the Instituto Colombiano de Crédito Educativo y Estudios Técnicos en el Exterior (ICETEX), are suitable to be analyzed from the conceptual and methodological approach proposed herein. Keywords: complexity; public policies; government agenda; public problems; actors; decisionmaking process
\end{abstract}

\section{Problemas, atores e decisões nas politicas públicas. Quadro de análise para o estudo dos programas de crédito educativo na Colômbia}

\section{Resumo}

Este artigo propõe um quadro de análise para o estudo de processos decisionais associados com a implementação de crédito educativo na Colômbia, como dispositivo de política pública que visa promover o acesso ao ensino superior. O enfoque conceitual e a proposta metodológica aqui apresentada contêm elementos do paradigma da complexidade e sua relevância na análise de política pública. Este quadro de análise concentra-se em estudar as relações existentes entre as correntes de atores, problemas e decisões de política, em cuja inter-relação surgem elementos explicativos sobre a maneira em que o processo de making policy é conduzido, desde a elaboração do problema para a tomada de decisões. O trabalho conclui com um esboço sobre o jeito em que a elaboração e implementação dos programas de financiamento educativo na Colômbia, impulsionados pelo Instituto Colombiano de Crédito Educativo e Estudos Técnicos no Exterior (ICETEX), reúnem as condições a serem analisadas desde a perspectiva conceitual e metodológica proposta no artigo.

Palavras-chave: complexidade; políticas públicas; agenda de governo; problemas públicos; atores; processo decisional 


\section{Introducción}

Las políticas públicas son acciones que aspiran a transformar la realidad; son las acciones de la politica llevadas a cabo para modelar una realidad imperfecta, intentando que se acerque a una visión idealizada; son los instrumentos de mejoramiento que la política tiene a su disposición para hacer efectivas sus promesas (Brugué-Torruela, 2014).

Los análisis de policy constituyen un ejercicio riguroso y complejo; si se considera secuencial y cronológicamente, una política pública pasa de la identificación de una situación insatisfactoria a la definición del problema y la subsecuente definición de alternativas de intervención. Sin embargo, analíticamente, las tres etapas son interdependientes. Con mayor o menor frecuencia, incorporar una problemática en la agenda de gobierno implica preconcebir modelos mentales que dan forma a la definición del problema aceptable para la sociedad y, sobre todo, tratable para el gobierno. De este modo, los procesos decisionales terminan adaptando los problemas de la agenda politica a las tecnologías de intervención disponibles, o ajustando, con ciertas modificaciones, las estrategias de intervención existentes a características de los problemas que son proclives de intervenir.

En el campo de las políticas de educación superior existe consenso de manera casi generalizada en que el acceso inequitativo constituye un agudo problema social, no solo por su incidencia en el cierre de brechas de ingresos económicos y calidad de vida, sino por su aporte a la redistribución de capitales y el acceso a mejores oportunidades de empleo. En este marco, Colombia ha sido pionera en el desarrollo de estrategias de crédito educativo, gracias a la creación del Instituto Colombiano de Crédito Educativo y Estudios Técnicos en el Exterior (ICETEX), que con el transcurso de los años ha ganado un papel protagónico y una creciente influencia en el escenario de las políticas públicas para el fomento del acceso y la permanencia en la educación superior, y en la gestión de becas para el perfeccionamiento de estudios de postgrado. 
A lo largo de los años, el Instituto ha promovido el imaginario del crédito como instrumento para ampliar el acceso a la educación superior a jóvenes provenientes de sectores incapaces de comprar educación superior privada y a personas que, sin disponer del total del dinero para financiar su matrícula, acuden al mecanismo del crédito para diferir el valor con préstamos a mediano plazo.

Pese a la importancia de los créditos educativos en el escenario de politicas educativas nacionales, no existe un marco analítico que alimente un estudio de las diferentes etapas que transcurren desde su elaboración hasta su puesta en marcha. En este artículo nos interesa construir un marco de referencia analítica que permita analizar el proceso de elaboración e implementación de las políticas de crédito educativo para fomentar el acceso de los sectores de escasos recursos a la educación superior, basado en el paradigma de la complejidad aplicado a las políticas públicas. Ello es, desde un enfoque en el que interactúan de manera más o menos independiente, corrientes de actores, problemas, decisiones y oportunidades de elección (Dente y Subirats, 2014).

Este propósito suscita varios interrogantes analíticos. En primer lugar, interesa comprender la elaboración del problema público que da origen a los créditos como dispositivo de política pública para fomentar el acceso a la educación superior. De acuerdo con Hogwood y Gunn (1984), la definición del problema encierra los procesos por los cuales una cuestión incluida en la agenda de la política pública es estudiada, explorada, organizada y posiblemente cuantificada por los interesados, quienes no raramente actúan en el marco de una definición de autoridad, aceptable de manera provisional en términos de sus probables causas, componentes y consecuencias.

Esta definición nos traslada a un segundo planteamiento relacionado con los actores inmersos en la arena de la politica educativa y los escenarios en los que generan sus propuestas. Serán actores de política pública todos aquellos que participan o influencian los procesos de policy, incluyendo a quienes actúan por omisión, pues 
en esta conducta pueden encontrarse factores explicativos de una decisión de política.

El estudio de los actores cobra importancia en un análisis de política pública en la medida en que, por lo general, quien incorpora la definición aceptada del problema público, suele ejercer mayor influencia en el proceso de búsqueda y asignación de soluciones (Aguilar, 1993). De esta manera, la forma en que se define un asunto público condiciona las opciones de acción y las estrategias de implementación de una política.

Un tercer punto de análisis tiene que ver con el proceso de toma de decisiones. Entendemos el estudio de las decisiones como el conjunto de criterios y procesos mediante los que se escoge una estrategia o curso de acción en un abanico más amplio de propuestas (Dente y Subirats, 2014). Con frecuencia, el estudio de las decisiones guarda una estrecha relación con el análisis de los actores de política, pues el rol y los intereses que cada uno maneja en la arena de la política pública, constituyen elementos explicativos de los procesos decisionales.

Con base en estos tres planteamientos, el presente artículo ha sido organizado en cinco secciones. La primera aborda la relación entre el análisis de política pública, el paradigma de la complejidad y la teoría general de sistemas. La segunda aborda la cuestión de los problemas públicos y su incorporación a la agenda de gobierno, enfatizando en la multidimensionalidad de los asuntos de política y su relación con los intereses y objetivos de los participantes. La tercera sección brinda elementos para la identificación y perfilamiento de los actores que intervienen en cada uno de los procesos de política pública. La tipología aquí propuesta parte de la identificación de los roles que asume cada actor y de los recursos con los que cuenta para ejercer influencia o autoridad en cada una de las etapas del proceso. La cuarta parte aborda un modelo de análisis decisional a través del cual se argumenta que los actores de política pública suelen tener preferencia por situaciones problemáticas difusas y tecnologías de intervención poco claras que, en consecuencia, afectan negativamente la efectividad de una política pública. La parte final brinda una caracterización del Instituto de 
Crédito Educativo y Estudios Técnicos en el Exterior (ICETEX), que justifica la implementación del modelo analítico aquí propuesto en el marco de sus créditos educativos como repuesta de politica educativa para el fomento del acceso a la educación superior.

\section{Complejidad y politicas públicas}

El análisis de policy que se propone en este artículo utiliza como perspectiva teórica y propuesta metodológica el análisis de la teoría general de sistemas, asociado al paradigma de la complejidad, para explicar y analizar algunos de los principales aspectos de la compleja red de relaciones que integran el escenario de las politicas públicas. La hipótesis subyacente es que mediante este enfoque resulta posible identificar factores adicionales a los considerados desde la perspectiva secuencial de política pública.

Entendemos por política pública un complejo sistema conformado por subsistemas de politicas especializadas en diferentes campos de acción, que ofician como sistemas abiertos, ello es, sistemas integrados entre sí y con fuertes relaciones con el contexto circundante (Bertalanffy, 1968/1987), cuyas dinámicas influyen, en mayor o menor medida, los procesos de política.

La interrelación entre los participantes de la política, sus recursos, intereses y el espacio donde se ponen en juego, constituyen elementos que pueden explicar el curso de las decisiones y los resultados de una política pública. Con esto ponemos el acento en que los análisis de política pública son un ejercicio difícil de reducir a una perspectiva estructuralista.

Los procesos de la política no pueden ser explicados en su totalidad desde una perspectiva que considere sus componentes de manera aislada, o que estudie de modo autónomo cada una de sus fases. Asistimos, pues, a un nuevo paradigma de análisis que, al concentrar su foco de estudio en el contexto donde interactúan los elementos 
constitutivos de la política, y en las condiciones y circunstancias donde estos se manifiestan, no puede escaparse de una perspectiva holística e integradora (Caro, 2002).

En este sentido, desde la perspectiva de la complejidad, el conjunto de procesos que estructuran las políticas públicas abarca un sistema donde interactúan, con mayor o menor independencia, corrientes de actores, problemas, decisiones y oportunidades de elección. Su estudio se entiende, en esta medida, como un proceso complejo sin principio, ni final, y cuyos límites permanecen, hasta cierto punto, inciertos. Esta red compleja de fuerzas cuyos límites permanecen difusos produce, en consecuencia, y de alguna manera, un efecto llamado política pública (Lindblom, 1991).

\section{La corriente de los problemas públicos y la agenda de gobierno}

El análisis de los problemas de politica pública constituye un elemento central en un análisis de policy. Este ejercicio permite identificar quiénes legitiman y definen los problemas públicos y qué grupos y organizaciones tienen la fuerza suficiente para que un problema social trascienda al interés público. Dicho de otro modo, evidencia la estructura de poder que domina el proceso de elaboración de una política.

Con frecuencia se afirma que los problemas de la política se definen en medio de la discrepancia entre lo que debería ser y lo que realmente está ocurriendo. Los problemas (o asuntos) de la política constituyen situaciones problemáticas que, al afectar a determinado sector de la sociedad, llaman la atención del gobierno, que despliega cursos de acción para intervenir su estructura general, sus causas o consecuencias.

No obstante, no todos los problemas llaman la atención de la política, pues ni todos los problemas son públicos, ni todos conciernen a esta. En otras palabras, si una situación particular o un conjunto de 
circunstancias constituyen un problema y es propiamente un asunto de "interés público", es algo que depende de creencias, valoraciones y juegos de poder de los actores y no de los hechos mismos.

Para que un problema constituya un asunto público, requiere cumplir tres condiciones: 1) Que sea objeto de atención amplia o al menos de amplio conocimiento del público, ello es, que afecte negativamente a un grupo considerable o influyente de la sociedad; 2) Que una buena parte del público considere que se requiere algún tipo de acción; y 3) Que, a los ojos de los miembros de la comunidad, la acción sea competencia de alguna entidad gubernamental (Aguilar, 1993).

En este sentido, el nivel de conflictividad de un problema es un factor clave que bien puede favorecer o reducir su expansión o difusión, y, en consecuencia, su incorporación en la agenda del gobierno. Problemas con graves consecuencias que no logran trascender de la difusión en una pequeña escala, por lo general quedan circunscritos en su localidad y pasan desapercibidos por el gobierno. Solo aquellas demandas potencialmente conflictivas, ya sea por sus consecuencias o por involucrar un número amplio o influyente de actores, son candidatos efectivos para suscitar una estrategia de intervención.

Dado que los límites de un asunto público no son completamente claros, los analistas utilizan el término "formación de agenda pública", para referirse al conjunto de problemas y sus características, que el gobierno selecciona para intervenir (Montecinos, 2007). Y aunque los problemas cumplan con los tres criterios enunciados, en la mayoría de oportunidades los criterios de esta elección varian en función de valores, intereses e incluso de la capacidad instalada con que cuentan los gobiernos para adelantar cursos de acción.

Un problema puede acceder a la agenda de las políticas públicas de tres maneras: no segmentado, de manera jerárquica o de manera especializada (Cohen, March y Olsen, 1972). En el primero, cualquier problema tiene acceso a las soluciones. En el segundo, los problemas de mayor relevancia para los actores pueden ser intervenidos con más de una alternativa de política, de la misma forma en que tienen mayor 
preferencia para ser acuñados a las tecnologías de intervención que gozan de un reconocimiento más amplio dentro de la organización. En el tercero, cada problema tiene acceso a una elección y cada elección es accesible solo a dos problemas, ello es, elecciones especializadas en los tipos de problemas que pueden guardar alguna relación con sus componentes y formas de intervención.

En suma, los problemas que ingresan a la agenda son construcciones sociales que plasman ciertas concepciones particulares de la realidad de quienes ejercen influencia y/o autoridad a lo largo del proceso (Aguilar, 1993). Esto quiere decir que la conformación de la agenda de los problemas públicos requiere de la comprensión de dos categorias analíticas, en cuyo interjuego se pueden reconocer elementos explicativos sobre la manera en que los problemas públicos ingresan a su estructura y, en consecuencia, se traducen en cursos de acción; hablamos de la influencia y la autoridad.

La influencia hace referencia al atributo con que cuentan los actores cuando no están respaldados por una estructura jerárquica que les confiera autoridad en el proceso. La influencia es un elemento que permite visibilizar un problema público y expandirlo lo suficiente hasta ser considerado en la agenda. La reunión de un conjunto de actores con intereses, creencias y objetivos similares en cuanto a un asunto público se denomina grupo de interés. Y los grupos de interés configuran fuertes alianzas para incorporar un problema a la agenda de los gobiernos.

La dimensión de la autoridad representa la manera en que, basados en una organización vertical, ciertos actores ubicados en la parte superior de la pirámide cuentan con atributos administrativos y legales que les brindan facultades para determinar asuntos públicos y tomar decisiones.

Por lo general se afirma que las comunidades afectadas por un problema público configuran escenarios activos de lucha entre juegos de influencia y poder, mientras que los gobiernos suelen comportarse pasiva y reactivamente, dejando que las cuestiones se configuren y 
definan dentro del juego de las fuerzas sociales, e intervienen estratégicamente en el proceso influyendo la construcción del problema a la luz de sus posibilidades reales de intervención, es decir, a través de los dispositivos con que cuentan para intervenir la problemática (Aguilar, 1993, p. 37).

La trayectoria de un problema desde su nacimiento en un contexto dado, hasta su incorporación en la agenda, es en realidad una constante confrontación entre actores y decisiones; y el resultado suele ser la aceptación colectiva de que existe un problema con una línea gruesa compartida por los participantes, y que efectivamente su intervención compete al gobierno, aunque en realidad persista de fondo un desacuerdo sobre la composición del asunto, sus alcances, sus causas, sus consecuencias o la manera de abordarlo (Aguilar, 1993).

\section{La corriente de los actores de la política pública}

De la mano con el estudio de la elaboración e incorporación de un asunto público en la agenda de gobierno, el estudio de los actores constituye un factor central en todo el análisis de making policy. Analizar los actores implica reconocer los valores, creencias y modelos mentales que dan lugar a definiciones específicas de un problema de política y al perfilamiento de estrategias y tácticas que definen su margen de acción.

Como veremos más adelante, la lista de participantes a lo largo de los procesos de política incluye actores formalmente vinculados a los procesos de formulación de política - políticos y burócratas, entre ellos-, como actores informales que, sin que la politica pública los afecte directamente, persiguen intereses particulares. Por lo general, buena parte de la ciudadanía no suele participar en los procesos de making policy, y cuando lo hace, usualmente su papel no es determinante en la decisión final. Los procesos de política pública en las democracias modernas suelen ser más complicados que en los países con dictadura, pues en las primeras hay una mayor pluralidad 
de actores debido a la separación de poderes, la existencia de mecanismos de supervisión de la acción política y el principio de control y equilibrio que rigen los sistemas democráticos (Lindblom, 1991).

En todo caso, los actores de la política pública están inmersos en un subsistema de relaciones donde los objetivos que persiguen no solo pueden comprenderse por los flujos de información que intercambian con otros participantes, sino también por la relación que estos mantienen con el contexto sociopolítico donde se sitúa el proceso.

A diferencia de la red de actores, que se concentra en los intercambios de información y recursos entre los participantes, el subsistema contribuye a entender los juegos de poder en los procesos de la política. El concepto de subsistema quiere mostrar que la arena de la política pública se divide en campos específicos de acción de política pública, llamados subsistemas de política, donde los actores concentran diferentes flujos y cargas de autoridad e influencia, lo que termina favoreciendo una organización vertical en la toma de decisiones. En la medida en que existen campos especializados de política, los actores son obligados a adoptar dinámicas similares de especialización. A este respecto, Aguilar (1993, p. 50) sostiene:

La configuración del poder en los subsistemas es muy variada. Algunos subsistemas están bajo el dominio de uno o pocos actores muy poderosos; en otros el poder es bastante difuso. Manifiestan poseer un orden normativo con símbolos, mitos, rituales y hasta lenguajes singulares, que no hacen sino reflejar la realidad intersubjetiva de sus miembros y su acuerdo sobre lo que en su opinión es importante, deseable o correcto. Se integran con actores muy diversos: instituciones, organizaciones, grupos e individuos. Todos ellos articulados por el hecho de que comparten intereses importantes en una política particular.

En suma, todo individuo o grupo social vinculado con el problema de política es un actor potencial en la arena de la política pública, independientemente de su margen de acción e incluso si este 
es incapaz de emprender acciones concretas durante alguna de las fases del proceso.

Rechazamos la idea de denominar actores únicamente a los sujetos (individuales o colectivos) que ejercen influencia en un proceso de policy, en la medida en que la pasividad de un actor (voluntaria o involuntaria) puede constituir un factor explicativo de la selección de una determinada política frente a otras posibles (Knoepfel et al., 2007). En este sentido, todos los actores cuentan con un margen de maniobra en la arena de las políticas que puede ser mayor o menor de acuerdo a los objetivos que persigue, al rol que desempeña y de los recursos que tiene a su alcance para influir en el proceso.

En general un actor puede perseguir dos tipos de objetivos; cada participante puede privilegiar sus acciones con base en uno de estos tipos o combinarlos. Llamamos a estos objetivos de contenido cuando tienen que ver con las preferencias relativas al problema y las soluciones, y de proceso cuando están asociados a las consecuencias que la definición aceptada del problema y los efectos de la política seleccionada tienen sobre sus recursos y sobre los demás participantes.

Por lo general, los objetivos de proceso guardan una estrecha relación con las dinámicas de poder y autoridad que se dan dentro de los subsistemas de la política pública. Mientras que todos los actores, independientemente de su rol o su ubicación en el proceso de política, persiguen en alguna medida objetivos de contenido, los objetivos de proceso constituyen una expresión de la manera en que cada actor desea tomar cierto protagonismo en el proceso (Dente y Subirats, 2014).

Como vemos, si el análisis de política se concentrara de manera exclusiva en los objetivos de contenido, el ejercicio se orientaría únicamente a la identificación de las percepciones de los actores alrededor del problema y las soluciones. Cuando incorporamos los objetivos de proceso, el ejercicio se complejiza en la medida en que reta al analista a estudiar el juego de poder escondido en la arena de la política. Ahora bien, cuando además de considerar las implicaciones de ambos tipos de objetivos, involucramos en el análisis la forma en que el 
contexto sociopolítico influye a lo largo del proceso de making policy, evidenciamos las relaciones complejas que caracterizan los análisis de política pública.

Ahora bien, partir del hecho de que todos los actores persiguen en mayor o menor medida objetivos de proceso o de contenido, implica aceptar que todos son de cierto modo racionales, en el sentido en que, para legitimar sus argumentos, defender una posición y perseguir determinados objetivos, cada participante pone en juego los recursos que tiene a su disposición, que pueden involucrar desde potestades legales y políticas hasta recursos económicos y cognitivos.

Dente y Subirats (2014) distinguen cuatro tipos de recursos que pueden explicar la diversidad de roles y el nivel de influencia o autoridad que estos ejercen en los procesos de politica:

- Recursos políticos: consisten en la cantidad de consenso que un actor es capaz de poner en movimiento. Tal consenso puede referirse a la población entera o a grupos sociales determinados, implicados en las diversas políticas públicas.

- Recursos económicos y financieros: consisten en la capacidad de movilizar recursos económicos de cualquier tipo para modificar o influenciar los comportamientos de los otros actores.

- Recursos legales: consisten en las potestades, o posiciones de ventaja, que las normas jurídicas, y en general los dispositivos legislativos, administrativos y legales, atribuyen a algunos sujetos.

- Recursos cognitivos: consisten en la disponibilidad de informaciones o de modelos interpretativos relevantes en relación con la adopción de una decisión.

A raíz de la racionalidad y los recursos de cada participante, distinguimos cinco categorias de actores: actores políticos, actores burocráticos, beneficiarios finales, población objetivo y actores terciarios. 
Los actores políticos justifican su participación en los procesos de política debido a que representan a los ciudadanos, al gozar de un apoyo masivo dado por la manera en que llegaron al poder. El supuesto de legitimidad que se esconde tras la participación de estos actores implica que, en un sistema democrático, no se pueden tomar decisiones sin el consenso popular. Esto implica que, cuanto más alta sea la cuota de consenso que dicho sujeto se atribuye, más alta será la influencia que este ejerza en el proceso de política pública (Dente y Subirats, 2014).

Los actores burocráticos son aquellos sujetos que basan sus lógicas de acción en recursos legales (leyes, normas, decretos) que les atribuyen potestades específicas en un proceso de política pública. Con mayor o menor frecuencia, la especialización por campos de política pública es visible a través de la manera en que se encuentran organizadas las entidades e instituciones que conforman los Estados modernos. La hipótesis de intervención que apoya esta categoría de actores se basa en que, con frecuencia, la organización estatal requiere, para hacer más eficientes sus procesos, diferenciar con la mayor claridad posible qué actores asumen determinadas responsabilidades y qué mecanismos tienen a su disposición para ejercerlas. Se trata del reemplazo de la legitimidad tradicional por la legitimidad legal-racional en el sentido weberiano (Weber, 1996).

La población objetivo reúne al conjunto de la sociedad cuyo comportamiento es considerado, politicamente, como la causa directa o indirecta del problema, y donde se encuentran factores explicativos de un problema de política pública. Los actores implicados en este grupo son, por lo general, difusos, porque su inclusión en la categoría depende esencialmente de la elaboración mental del problema y del nivel de consenso que logren los actores al momento de incorporar el asunto público a la agenda del gobierno.

Los beneficiarios finales son aquellos actores que, siendo los principales afectados por el problema, se proyectan como los principales beneficiados de la política pública. Sin embargo, es importante aclarar que los beneficios no son exclusivos de este grupo cuando los 
actores persiguen objetivos de proceso. En muchos casos la elaboración de una política pública termina ajustándose a los beneficios que esta pueda traer a los demás implicados del proceso.

Finalmente, la categoría de actores terciarios o informales se refiere al conjunto de participantes que, siendo en su mayoría privados y sin que la política pública les esté directamente destinada o las estructuras legales y burocráticas los involucren directamente, actúan considerando la probabilidad de cierto impacto de los resultados de la estrategia de intervención en ellos o sus intereses.

Eneste último grupo encontramos: 1) Actores con intereses generales que, sin estar dotados de legitimidad política o legal, representan a sujetos o intereses que no pueden defenderse por ellos mismos, y que por lo tanto no son capaces de actuar en primera persona; 2) Actores con intereses especiales, o dicho de otra manera, actores con objetivos de proceso que actúan porque la intervención puede beneficiar sus intereses; 3) Intelectuales expertos, sujetos que al poseer un considerable volumen de recursos cognitivos cuentan con la legitimidad necesaria para intervenir en los asuntos de política.

Los intelectuales expertos condicionan en cierta medida la problematización de los asuntos de la política, pero son los grupos de interés en cualquiera de las dimensiones de la politica, los que terminan influyendo en la problematización y la respuesta a determinados asuntos. Los expertos responden, finalmente, a los asuntos que los grupos de interés logran posicionar en la agenda pública (Aguilar, 1993).

Los grupos de interés representan a un conjunto de actores que, actuando de manera grupal, tienen mayores probabilidades de incidir en los procesos de politica que de manera individual. Pese a que tradicionalmente los grupos de interés constituyen categorías analíticas de estudios micropolíticos, por lo general, dinámicas asociadas a la agrupación de actores con intereses similares suceden a lo largo de los procesos de elaboración de política en la dimensión de las comunidades como en la de los actores que dicen e implementan. Con frecuencia se crean coaliciones de actores e instituciones en aras de favorecer, bien 
sea la elección de un problema específico de política de acuerdo a sus modelos mentales, o la elección de un curso de acción que beneficie sus intereses.

\section{La corriente de las decisiones: la papelera de reciclaje como modelo decisional de politica pública}

La complejidad de las políticas públicas se traduce en un conjunto de relaciones interconectadas detrás de cada decisión de política pública. Desde el enfoque de la complejidad, es posible comprender las organizaciones como una estructura donde se entrelazan actores, problemas y decisiones en un orden no del todo jerárquico ni racional.

Esta perspectiva se asemeja en mucho a los modelos de las "anarquías organizadas" que deciden desde el modelo de la papelera de reciclaje $^{3}$, en el que por lo general se adaptan situaciones problemáticas a cursos de acción ya constituidos. La característica central de este modelo es el desacoplamiento entre los problemas y las soluciones.

Esta mirada concentra el análisis del proceso de toma de decisiones en la manera en que las organizaciones optan por determinados cursos de acción, no por una lógica racional, sino por un conjunto de factores que varian en función de los actores con influencia en el proceso, de los intereses y de las alternativas al alcance para intervenir el problema.

Sin intención de generalizar, este modelo explicativo de toma de decisiones puede ser usado para entender la forma en que se gestan las políticas públicas y en la que progresivamente algunas se desechan, otras se fortalecen y otras son adaptadas a las necesidades del contexto. Así mismo, este modelo puede ser utilizado para comprender algunas consecuencias de la estructura de los procesos decisorios de políticas educativas en el país.

3 En su idioma original: Garbage Can Model. 
En contextos donde existe una considerable pluralidad de actores que nutren la complejidad de un problema de política pública, suele hablarse de modelos de toma de decisiones basados en la lógica de las anarquías organizadas. Estas son la representación de un modelo de toma de decisiones poco racional, con preferencias problemáticas, donde las tecnologías de intervención no son completamente claras y los flujos de relación entre actores resultan variantes de acuerdo a sus intereses, los recursos y el momento en que se discute un determinado problema de política pública.

Este modelo irracional de toma de decisiones suele representarse bajo la metáfora de los botes o papeleras de reciclaje. En estas, las organizaciones arrojan problemáticas, esperando que, al interior, el problema se una, de una u otra forma, con una solución que espera dentro del bote a ser usada para fines ligeramente coincidentes con sus alcances. Las papeleras representan una colección de decisiones para superar problemas y asuntos públicos, que buscan situaciones de decisión en las cuales puedan ser puestas en práctica.

Cohen, March y Olsen (1972) asocian el concepto del modelo de decisiones bajo la perspectiva de los botes de basura a la forma en que funcionan y se organizan las anarquías organizadas. Estas suelen tener preferencias por problemas difusos y por alternativas de solución igualmente ambiguas.

Las preferencias problemáticas hacen referencia a que las organizaciones operan sobre la base de problemas mal estructurados, que confunden usualmente las causas del problema con sus efectos, y en consecuencia terminan organizando agendas de política pública, más similares a una colección dispersa de ideas que a una estructura coherente de problemáticas a las cuales atacar. Las tecnologías de intervención que se despliegan con esta lógica terminan comprobando las preferencias de quienes deciden, donde se sobrepone el deseo de usar determinados mecanismos de intervención sobre el objetivo de combatir el problema. 
La ambigüedad de los problemas de politica que hemos enunciado conduce a la selección de tecnologias de intervención poco claras. Esta baja claridad de los mecanismos que componen una estrategia, generalmente conlleva a que los actores involucrados en el proceso no comprendan por completo las relaciones que se tejen entre el modelo de intervención y el problema al que se acuña, impidiendo que se defina con claridad el efecto que se quiere lograr a través de la puesta en marcha de una estrategia de política pública. En sintesis, las tecnologías poco claras terminan actuando sobre la base del ensayo y el error, lo que implica que el proceso incremental de la politica obligue a los decisores a formular y reformular la estrategia de intervención, o con mayor frecuencia, a re-elaborar el problema en función de la tecnología que se está poniendo en práctica.

La ambigüedad de los problemas y las tecnologías de intervención está estrechamente vinculada con la variación en cantidad y dedicación de los actores que participan en el proceso decisional. Como resultado, los límites de la arena de política pública no son claros, y las decisiones varian en función de los actores que encabezan las instituciones responsables del abordaje de un problema de política pública en determinado momento.

Este comportamiento está estrechamente ligado con lo que Cohen, March y Olsen (1972) denominan como dos problemas críticos de las anarquías organizadas. El primero se refiere a la manera en la cual las organizaciones toman decisiones sin tener metas consistentes y compartidas. Dada la ambigüedad de las metas, los procesos de elección no se llevan en el marco de negociaciones entre todas las partes del problema.

El segundo se refiere a la manera en la que los flujos de participación de los actores en los procesos de política pública varian en función del tiempo y los intereses. Esto tiene que ver con la cuestión de cómo determinados actores ejercen mayor o menor influencia o autoridad en ciertos momentos del proceso de elaboración e implementación de políticas públicas y cómo este fenómeno constituye un 
factor explicativo de la manera en que se eligen determinados cursos de acción sobre una oferta más amplia de alternativas de intervención.

Tal como advertimos previamente, las percepciones y elaboraciones mentales de un problema de política pública y su estrecha relación con los actores, sus recursos y su adscripción a uno o más roles dentro del proceso constituyen factores explicativos del proceso decisional de una política pública.

La lógica de las decisiones comprende que dentro de una organización pueden darse tres tipos: decisiones no segmentadas, decisiones jerárquicas y decisiones especializadas. En el primer tipo, cualquier actor de política pública puede participar en la elección de un curso de acción. En la segunda, los actores y las decisiones están organizados de manera jerárquica de acuerdo con los recursos que tienen a su alcance. En este sentido, las decisiones "importantes" están reservadas para los actores con mayores recursos a su disposición, de la misma forma en que los actores con mayor autoridad pueden participar en más de un proceso decisorio. En el tercer tipo, la estructura está diseñada de manera tal que los actores responsables de la decisión se especializan en las elecciones que ellos deben atender de acuerdo con las atribuciones legales y administrativas que le permite la arena de las políticas públicas.

Frecuentemente las decisiones no se toman de manera exclusiva con base en uno de estos modelos, sino que resultan de un proceso de combinación entre los tres. Así, las decisiones se pueden tomar a través de tres formas:

- Por propósito: se refieren a las elecciones que resuelven los problemas en determinados periodos de tiempo que pueden variar de acuerdo con las dinámicas específicas de los problemas. Este tipo de decisión es el característico de los procesos racionales de política pública.

- Por descuido: cuando una solución termina disminuyendo uno de los componentes de un problema, pese a que esta esté ligada a 
otra problemática. La multidimensionalidad de los problemas de política pública contribuye a que sucedan tal tipo de situaciones.

- Por traslape $e^{4}$ : cuando la intervención a un problema se caracteriza por la llegada de una nueva política que, de manera más o menos racional, fue pensada para intervenir uno o más componentes del problema en cuestión. En este caso, sucede un proceso de traslape en el que una solución muta o es reemplazada por otra más llamativa. En esta lógica los problemas son añadidos automáticamente a nuevas tecnologías.

Si bien este modelo de toma de decisiones no resuelve completamente los problemas, sí constituye un esquema analítico para comprender la forma en que se elaboran los problemas de la política pública y su intrínseca relación con la gama de elecciones de intervención, aun cuando las organizaciones se caractericen por una alta ambigüedad en los problemas, los actores, las decisiones y las oportunidades de elección.

\section{Claves para el análisis del crédito educativo como dispositivo de política pública en Colombia}

El ICETEX es la entidad colombiana responsable del fomento y promoción del desarrollo educativo y cultural de la nación a través de préstamos personales y otras ayudas financieras a los estudiantes y sus familias (MEN, 1968, Decreto 3155, Art. 2). El Instituto se crea como una entidad anexa al Ministerio de Educación Nacional mediante el Decreto 2586 de 1950. Denominado Instituto de Estudios Técnicos en el Exterior, se funda con el objetivo de diseñar, coordinar e implementar las estrategias de fomento de acceso a la educación superior en materia de estudios técnicos y profesionales, financiados por medio de recursos estatales y extranjeros (MEN, 1950, Decreto 2586, Art. $1^{\circ}$ ).

\footnotetext{
4 En su traducción original al español: decisiones tomadas al vuelo.
} 
En 1968, el ICETEX se transforma en el Instituto Colombiano de Crédito Educativo y Estudios Técnicos en el Exterior mediante el Decreto 3155 de 1968, que lo separa del Ministerio de Educación Nacional y lo transforma en "un establecimiento público, esto es, con personería jurídica, autonomía administrativa y patrimonio independiente" (MEN, 1968, Decreto 3155, Art. $1^{\circ}$ ). Esta transformación fortalece las responsabilidades del Instituto frente al fomento y promoción del desarrollo educativo y cultural en el país; concediéndole el monopolio del otorgamiento de créditos personales y otras ayudas financieras, apoyados con recursos públicos, a los estudiantes, para el fortalecimiento del capital humano del país (MEN, 1968, Decreto 3155, Art. $2^{\circ}$ ).

En la década de los noventa, la Ley 30 de 1992, reafirma la participación estratégica del Instituto como aliado estratégico para el cumplimiento de las metas del sector educativo, al convertirlo en el principal responsable de la provisión y mantenimiento de "un adecuado financiamiento de las matrículas y sostenimiento de los estudiantes [de la educación superior]" (MEN, 1992, Ley 30, Art. 112). Desde entonces, el ICETEX es el garante de los préstamos otorgados por el sector financiero a los estudiantes de Educación Superior de escasos recursos económicos, y conserva el monopolio de los recursos fiscales destinados a becas, o a créditos educativos universitarios en Colombia, que adquirió desde su nacimiento (MEN, 1992, Ley 30, Arts. $113^{\circ}$ y $114^{\circ}$ ).

En 2005, el Instituto se transforma en una entidad financiera de naturaleza especial cuyo propósito es el fomento social de la educación superior, priorizando sus acciones en la población de bajos recursos económicos y los estudiantes con mérito académico en todos los estratos, a través de mecanismos financieros para promover el acceso y la permanencia en la educación superior (MEN, 2005, Ley 1002, Art. $2^{\circ}$ ). Desde entonces, si bien la mayor parte de la financiación de la entidad se realiza con fondos de la nación, ICETEX tiene abiertos convenios con entidades territoriales (alcaldías y gobernaciones), destinados a financiar en por lo menos un 50\% los créditos educativos de beneficiarios provenientes las regiones que aportan los recursos. 
Actualmente, el Instituto cuenta con líneas de crédito a corto, mediano y largo plazo, focalizadas en diferentes sectores socioeconómicos. Su principal línea de crédito a largo plazo, el Crédito ACCES (Acceso con Calidad a la Educación superior), recientemente redenominada Crédito de Largo Plazo "Tú Elijes", tiene el propósito de aumentar el acceso de los sectores económicamente más vulnerables y altos desempeños académicos, provenientes de los estratos $0,1,2$ y 3 .

El Crédito de Largo Plazo "Tú Elijes" financia la matrícula o el sostenimiento de estudiantes talentosos y de escasos recursos para que costeen económicamente sus estudios superiores (técnicos profesionales, tecnológicos, de ciclo complementario de escuelas normales superiores), con un crédito a largo plazo que comenzarán a pagar una vez finalicen el programa (ICETEX, 2007, 2011).

La línea de mediano plazo se focaliza por lo general en estudiantes de clases medias (estratos 3 y 4). En esta línea de crédito, los beneficiarios cancelan el 50\% del valor total del programa universitario durante el periodo en el que lo cursan, y por la vía del crédito educativo, financian el $50 \%$ restante, que empiezan a pagar en el momento en que obtienen el título correspondiente, por un tiempo no mayor a la duración del programa.

La línea de corto plazo financia el 100\% de la matrícula de cualquier estudiante, independientemente del estrato. El valor del préstamo, más los intereses generados, se cancelan durante el periodo de estudios del estudiante. Dada la inmediata capacidad de pago que deben tener los estudiantes y sus familias para acceder a esta linea, usualmente sus beneficiarios pertenecen a estratos socioeconómicos medios y altos.

La historia del Instituto y la manera en que ha tomado un papel central en la política de educación superior mediante el apoyo a sus líneas de crédito, justifican la necesidad de implementar un marco de análisis para comprender las dinámicas detrás de las politicas que ha venido impulsando. 
De un lado, los antecedentes dan cuenta de que el progresivo protagonismo del Instituto en el marco de formulación e implementación de estrategias para el fomento de acceso a la educación superior, lo convierten en un actor influyente en el escenario de las politicas educativas no solo por sus atributos legales, sino por el conjunto de recursos burocráticos e instituciones aliadas que lo respaldan ${ }^{5}$.

La manera en que se ha gestado la oferta de créditos educativos desde el ICETEX y el Ministerio de Educación Nacional ha contribuido a que la corriente de actores que han influido en el proceso sea fluida y variable y a que las estrategias de implementación apunten, por lo general, a un proceso incremental. Diferentes estudios (Bula et al., 2007; CEDE, 2008; OCDE y Banco Mundial, 2012; Sánchez y Velasco, 2014) han arrojado luces para identificar la variedad de facetas que puede tomar un actor de esta política y la mutación de intereses a lo largo del proceso. En muchos casos, aquellos actores que a lo largo del proceso decisional pueden considerarse actores burocráticos, adoptan el rol de población objetivo e incluso población beneficiaria en los procesos de implementación de la estrategia. Este análisis también permite identificar cómo los actores movilizan recursos para beneficiar determinadas concepciones del problema, y, en consecuencia, favorecer la intervención hacia ciertos elementos del problema y no hacia su integralidad.

De otro, pese a que el ICETEX fundamenta buena parte de su misión en combatir el problema del bajo acceso de los sectores pobres a la educación superior, diferentes corrientes de actores han debatido la efectividad de sus estrategias en este sentido, y han discutido, desde múltiples perspectivas conceptuales, las definiciones de la equidad en que estas se basan.

Un ejercicio riguroso de análisis documental arrojará que la definición que cada actor ofrece del problema guarda puntos de encuentro

En varios informes de organizaciones internacionales se ha resaltado la eficiente gestión del ICETEX, gracias a la manera en que ha crecido el número de beneficiarios en el transcurso de los años. Según información suministrada por Ministerio de Educación Nacional (2006) y OCDE (OCDE y Banco Mundial, 2012), el número de beneficiarios de Créditos ACCES pasó de 53.000 estudiantes en 2003 a más de 155.000 en 2011. Aunque la participación de las clases con menores recursos ha sido relativamente limitada, la mayor cantidad de estudiantes inscritos en el crédito educativo pertenece a los estratos 1,2 y 3 . 
y desencuentro con las manifestadas por otros participantes, enriqueciendo y complejizando una elaboración consensuada. En algunos casos, el problema se definirá de manera imprecisa, confundiendo sus causas con sus consecuencias (Bardach, 1993), lo que pese a dificultar el proceso decisional, enriquece la generación de propuestas de acción y legitima la perspectiva de la complejidad en las políticas públicas como marco de análisis de las estrategias de crédito educativo.

Frente al panorama de las decisiones, varios analistas han cuestionado la racionalidad tras la decisión de ubicar a los créditos como la punta de lanza para combatir la inequidad frente a otras posibles estrategias de intervención que podrían conducir al mismo objetivo (Gómez, 2001; Bula et al., 2007; CEDE, 2008; Gómez y Celis, 2009; Rodríguez, 2014). En este sentido, una perspectiva conceptual y metodológica para el estudio de los créditos educativos como tecnología de intervención puede basar su análisis en la confluencia de cuatro corrientes poco organizadas, que se mueven de manera independiente y que se relacionan de manera no del todo coherente entre ellas: los problemas, las soluciones, los actores y las oportunidades de elección (Aguilar, 1993).

En suma, en el estudio interrelacionado de estas cuatro corrientes se pueden encontrar elementos explicativos sobre la manera en que los créditos educativos han tomado progresivamente un papel protagónico en la política educativa colombiana para el acceso con equidad a la educación superior.

\section{Referencias}

Aguilar Villanueva, L. F. (1993). Estudio introductorio. En L. F. Aguilar Villanueva (Ed.), Problemas públicos y agenda de gobierno (pp. 15-72). México: Grupo Editorial Miguel Ángel Porrúa. 
Bardach, E. (1993). Problemas de la definición de problemas en el análisis de politicas. En L. F. Aguilar Villanueva (Ed.), Problemas públicos y agenda de gobierno (pp. 219-233). México: Grupo Editorial Miguel Ángel Porrúa.

Bertalanffy, L. (1968/1987). Teoría general de los sistemas [J. Almela, Trad.]. México: Fondo de Cultura Económica.

Brugué-Torruela, Q. (2014). Políticas públicas: entre la deliberación y el ejercicio de autoridad. Cuadernos de Gobierno y Administración Pública, 1(1), 37-55.

Bula, J. I. et al. (2007). Medición de Impacto del Crédito Educativo ACCES en la Cobertura, la Permanencia y el Desempeño Académico en la Educación Superior en Colombia. Bogotá: ICETEX.

Caro, A. (2002). El paradigma de la complejidad como salida de la crisis de la posmodernidad. Revista Discurso, 16-17, s.f.

Centro de Estudios sobre Desarrollo Económico (CEDE). (2008). Segunda Medición de Impacto del Crédito Educativo ACCES en el Acceso con Equidad, Permanencia, Desempeño Académico y Graduación en la Educación Superior en Colombia, 2003-2008. Bogotá: Universidad de los Andes - ICETEX.

Cohen, M., March, J. y Olsen, J. (1972). A Garbage Can Model of Organizational Choice. Administrative Science Quarterly, 17(1), 1-25.

Dente, B. y Subirats, J. (2014). Decisiones públicas: análisis y estudio de los procesos de decisión en políticas públicas. Barcelona: Ariel.

Gómez, V. M. (2001). La política de equidad social y la transformación de la educación superior. Documento presentado al Coloquio "Universidad, Estado, Sociedad". Bogotá, Colombia.

Gómez Campo, V. M. y Celis Giraldo, J. E. (2009). Crédito educativo, acciones afirmativas y equidad social en la educación superior en Colombia. Revista de Estudios Sociales, 33, 106-117. 
Hogwood, B. y Gunn, L. (1984). Policy Analysis for the Real World. England: Oxford University Press.

Instituto Colombiano de Crédito Educativo y Estudios en el Exterior (ICETEX). (2007). Estatutos del ICETEX. Bogotá: ICETEX.

Instituto Colombiano de Crédito Educativo y Estudios en el Exterior (ICETEX). (2011). Plan estratégico 2011-2014. Bogotá: ICETEX.

Knoepfel, P., Larrue, C., Varone, F. e Hinojosa, M. (2007). Hacia un modelo de análisis de politicas públicas operativo. Un enfoque basado en los actores, sus recursos y las instituciones. Ciencia Politica, 3, 6-29.

Lindblom, C. E. (1991). El proceso de elaboración de Políticas Públicas [E. Zapico Goñi, Trad.]. Madrid: Ministerio para las Administraciones Públicas.

Ministerio de Educación Nacional (MEN). (1950). Decreto 2586 de 1950. Recuperado de http:/ /www.mineducacion.gov.co/1621/article-103405.html

Ministerio de Educación Nacional (MEN). (1968). Decreto 3155 de 1968. Recuperado de http://www.mineducacion.gov.co/1621/articles-104219_ archivo_pdf.pdf

Ministerio de Educación Nacional (MEN). (1992). Ley 30 de 1992. Recuperado de http://www.mineducacion.gov.co/1621/article-86437.html

Ministerio de Educación Nacional (MEN). (2005). Ley 1002 de 2005. Recuperado de http://www.mineducacion.gov.co/1621/articles-146154_archivo_pdf. unknown

Ministerio de Educación Nacional (MEN). (2006). Cobertura: con calidad y equidad. Educación Superior Boletín Informativo, 6, 02-21.

Montecinos Montecinos, E. E. (2007). Límites del enfoque de las políticas públicas para definir un problema público. Cuadernos de administración, 20, 323-335. 
Organización para la Cooperación y el Desarrollo Económicos (OCDE) y Banco Mundial. (2012). La educación Superior en Colombia. Bogotá: Ministerio de Educación Nacional.

Rodriguez, J. A. (2014, febrero). Apuntes sobre las finanzas de la Universidad Nacional. Ponencia presentada en la Audiencia Pública "La Universidad Nacional: una asignatura pendiente del Gobierno". Universidad Nacional de Colombia, Bogotá.

Sánchez, F. y Velasco, T. (2014). ¿Los préstamos para educación superior mejoran el salario? Crédito Educativo y Mercado Laboral: una aproximación de regreso discontinua para Colombia. Investigación de Economía de la Educación, 9, 769-795.

Weber, M. (1996). El politico y el científico. Madrid: Alianza editorial.

\section{Cómo citar este artículo}

Pérez Enciso, H. A. (2017). Problemas, actores y decisiones en las políticas públicas. Marco de análisis para el estudio de los programas de crédito educativo en Colombia. Universitas Humanistica, 83, 247-273. http://dx.doi. org/10.11144/Javeriana.uh83.padp 